expensive, reactor scheme than the tokamak or stellarator with the possiblity of a smaller sized unit.

At the values of $\beta$ which are in principle possible in the RFP, the magnetic field will be relatively small and since the $B_{\Theta}$ field decreases outward with plasma radius as $1 / r$ the magnetic forces on external conductors can be quite modest. Since the aspect ratio is not restricted by stability considerations, an open structure with $R / a \cong 5-10$ can be used; this further eases the problems of magnetic forces and is advantageous for a reactor. If ignition by ohmic heating alone is possible, the complexity and cost of additional heating will be avoided, which is a major simplification.

Although as presently envisaged the RFP reactor would be pulsed, it is now clear that the magnetic field distribution is self-generating and this can lead to a quasi steady state operation. For full steady state operation, means must be found, as for all systems using transformer coupling, to drive the toroidal current continuously. Methods proposed for the tokamak using neutral beam or high frequency driven currents in principle might apply in a pinch, and will be investigated and tested at a more advanced stage of pinch development.

\section{REFERENCES}

1. Gibson A., Europhysics News 14 (1983) 4. 2. Baldwin D.E., Europhysics News 12 (1981) $8 / 9$.

3. Wobig H., Europhysics News 13 (1982) $8 / 9$.

\section{FURTHER READING}

- for early pinch work see Glasstone S., Lovberg R.H., Controlled Thermonuclear Reactions (D. Van Nostrand, Princeton, N.J.) 1960;

— for a summary of the results up to 1978 , see Ortolani S., Reversed Field Pinch Configuration, Nuclear Fusion 19 (1978) 535;

- for a general review of the RFP research up to 1979, see Bodin H.A.B. and Newton A.A., Reversed Field Pinch Research, Nuclear Fusion 20 (1980) 1255;

— for more updated reviews, see Bodin H.A.B. and Ortolani S., the Status of RFP Experiments, University of Padova Report UPee 82/09 (1982);

Dreicer H., Topics in Reversed FieldPinch Physics, Physica Scripta T2/2 (1982) 435; - for reports on progress in the major experiments, see the proceedings of the biennal IAEA Conferences on Plasma Physics and Controlled Nuclear Fusion Research;

- for a recent collection of papers on RFP theory and experiments see the proceedings of the Course and Workshop on Mirror Based and Field Reversed Approaches to Magnetic Fusion, Varenna, Sept. 7-17 (1983), to be published by the Commission of the European Communities.

\title{
The Seventh European Regional Astronomy Meeting
}

The Seventh European Regional Astronomy Meeting, organized by the European Physical Society and the International Astronomical Union, took place in Florence from December 12 - 16, 1983. It was cosponsored by the Italian Ministry of Education, Arcetri Astrophysical Observatory, the University of Florence, and the Italian Astronomical Society. The opening ceremony was quite spectacular since it was held in the old palace "Palazzo della Signoria", with trumpet players in Renaissance costumes behind the official table.

The EPS and IAU are interested in promoting scientific exchange between astronomers, astrophysicists and physicists, the borders between their disciplines often being difficult to define. One of the means of achieving this goal is to organize regular meetings on the European level: these meetings should also provide the young scientists with an opportunity to meet the more established, and to publicize their own work.

The IAU has been organizing European Regional Meetings for several years now;

- 1972, Athens: "Solar Activity and Related Interplanetary and Terrestrial Phenomena" - "Stars and the Milky Way System" _ "Galaxies and Relativistic Astrophysics".

— 1974, Trieste: "Stars and Stellar Evolution",

- 1975, Tbilisi: "Stars and Galaxies from Observational Points of View".

- 1978, Uppsala: "Stars and Star Systems".

— 1980, Liège: "Variability in Stars and Galaxies".

- 1982, Dubrovnik: "Sun and Planetary System".

The first six meetings were topical i.e. essentially devoted to one (sometimes very broad) subject whereas the seventh showed the result of a tighter collaboration between the EPS and the IAU, in the following sense: talks in any astrophysical discipline were accepted, and parallel sessions as well as invited talks were organized on topics at the frontier between astronomy and physics and/or in areas where new developments in physics are important for astrophysical research.

It is fair to say that this Seventh Regional Astronomy Meeting was a great success since it attracted over
400 participants, with the presentation of about 240 contributions. The invited papers will be published by the Societa Astronomica Italiana (Largo E. Fermi, 5, I-50125 Firenze).

During the last two days of the meeting, three full-day specialized sessions were organized on the following subjects:

- the NL-UK-USA Infrared Astronomy Satellite, IRAS;

- the European X-ray Satellite,

EXOSAT;

- Atomic and Molecular Physics for Astronomy.

\section{IRAS}

IRAS was launched on 25 January, 1983 , and ended its astronomical life on 22 November, 1983 when its coolant ran out, but in the course of the first six months, the spacecraft was able to view the entire celestial sphere. The cooled 22.4 in telescope mapped the area in bands centred around 12, 25, 60 and $100 \mu \mathrm{m}$ and made more detailed observations of several targets. There has been very little release of scientific data so far: first accounts are to appear in a series of papers in Astrophysical Journal Letters (March 1984), and a catalogue is scheduled for August-September 1984 (with positions and other information on more than 200000 far-infrared sources).

The specialized session organized in Florence gave the participants the occasion to listen to a series of talks which included several on comets and fast moving objects, the "minor planet" or burned out comet 1983 TB, a solar system dust ring, the "infrared cirrus" clouds (very cold dust at $\cong 35^{\circ} \mathrm{K}$, present even in the direction of the galactic poles), galactic cold dust (part of the "missing mass"?), stars with dust shells (ring of solid particles around Vega, older clouds at larger distances around Betelgeuse), the Crab Nebula, sources in Molecular Clouds, the Andromeda Galaxy M31, infrared galaxies (a huge number of them visible at the long wave bands), clusters and quasars, and the intriguing blank fields (IR sources with presently no optical counterpart). After these presentations of IRAS results, it was very clear that the astronomical community was anxiously waiting for the release of the first papers and of the catalogue since 
numerous exciting follow-up studies in various wavelength regions will have to be performed.

\section{EXOSAT}

The European X-ray satellite EXOSAT was launched at the end of May, 1983. Although some of the experiments on board caused problems during a few weeks, it now appears that many scientific results are becoming available, as was evident from the session organized on this satellite.

Thirty two contributed papers gave a good impression of the productivity and exciting new, although preliminary, results from the first months of EXOSAT in orbit. Particular interest was shown in the new spectroscopic data on several hot white dwarf stars and galactic binaries. The ability to carry out long uninterrupted observations and to observe in real time were evidently valuable features of the highly eccentric EXOSAT orbit. Coming only a few months after the start of the main observing programme, the session in Florence underlined the great potential of EXOSAT for European astronomers over the next few years.

Several international conferences are being organized in 1984 on the subject of X-ray astronomy: no doubt many results will be presented based on purely EXOSAT data or on simultaneous observations with e.g. EXOSAT, IUE and ground-based telescopes.

\section{Atomic and Molecular Physics for As- tronomy}

The aim of this meeting was to bring together "producers" and "users" of atomic and molecular data and theories.

According to many participants, it was a real success. Three invited papers and 25 original contributions were presented in one day; they were distributed among different sub-sections: term analysis and transition probabilities for atoms (7 papers), molecular physics (7), basic processes (2), line broadening (3), highly-ionized atoms (6). It is through this type of meeting that astrophysicists learn to understand the problems physicists encounter, while physicists better realize what are the needs for data and the role they play in astrophysics. Such goals have undoubtedly been achieved during the Florence specialized meeting: the present astronomical needs for accurate atomic and molecular data have been stressed, and many physicists have reported on new data which rapidly respond to the requests from astrophysicists.

The meeting on Atomic and Molecular Physics for Astronomy was in fact a very good example of cross-fertilization between physicists and astrophysicists and therefore fulfils one of the main aims of joint EPS/IAU ventures.

The Eighth European Regional Astronomy Meeting will be held in Toulouse, France, from September 17 - 21, 1984: it should, as in Florence, be open to all astrophysical disciplines, and have, in addition the following four specialized sessions:

- high spatial resolution in solar physics;

- new aspects of galaxy photometry;

- near molecular clouds;

- physical processes near the Eddington limit.

The Ninth Meeting is then scheduled for 1986, and it is hoped that it will take place as a "European forum": the loca- tion of this gathering is expected to be defined later this year, once offers from host cities or institutions have been received by the EPS or the IAU.

The success of the Florence Meeting has given a strong impetus towards the creation of a European Astronomical Society: there is no doubt that the European astronomical community has indeed become sufficiently strong and mature, and that the creation of such a society would be most timely and appropriate. Contacts to this effect are presently taking place, and the results will be discussed at the time of the Toulouse gathering (see also page 3).

\section{Jean-Pierre Swings}

(Secretary, Astronomy and

Astrophysics Divisional Board)

\section{CMD Section Committee Elections}

Following the recent elections within the Sections of the Condensed Matter Division, the basic Committees are made up as follows. These Committees will be electing their own Chairmen and coopting additional members:

\section{Low Temperature Section}

M. Krusius, University, Turku

F. Pobell, KFA, Jülich

H. Postma, University, Delft

F.B. Rasmussen, H.C. Ørsted Inst., Copenhagen

D. Thoulouze, CRTB-CNRS, Grenoble

W.F. Vinen, University, Birmingham

Macromolecular Physics Section

F. Baltà-Calleja, Inst. of Chem. Physics, Madrid

G. Bodor, Polymer Res. Inst. Budapest

A. Keller, University, Bristol

H.G. Kilian, University, Ulm

J.P. Mercier, High Polymer Lab.,

Louvain-la-Neuve

L. Monnerie, ESPCI, Paris

\section{Magnetism Section}

L.J. de Jongh, Kammerlingh Onnes

Laboratory, Leiden

R. Elliott, University, Oxford

J. Kaczer, Czechoslovak Acad. of Sciences, Prague

G.M. Kalvius, Tech. University, Munich

E. Karlsson, University, Uppsala

S. Methfessel, Ruhr Univ., Bochum

\section{Metals Section}

D. Bacon, University, Liverpool

K.H.J. Buschow, Philips' Res. Labs.,

Eindhoven

R. Cahn, University, Cambridge

O. Fischer, University, Geneva

J.P. Gaspard, University, Liège

E. Lüscher, Technical University, Munich
Semiconductors and Insulators Section J.W. Allen, University, St. Andrews

D. Bäuerle, University, Linz

F. Beleznay, Inst. for Technical Physics, Budapest

R. Blinc, Josef Stefan Inst., Ljublijana

H. Grimmeiss, University, Lund

G. Harbeke, RCA Labs., Zurich

Surfaces and Interfaces Section

G. Benedek, University, Milan

F. Koch, Tech. University, Munich

B.I. Lundqvist, Chalmers Univ. of

Technology, Göteborg

F. Saris, FOM Inst. for Atomic and

Molecular Physics, Amsterdam

H.C. Siegmann, ETH, Zurich

R.F. Willis, Cavendish Labs., Cambridge

\section{Elections \\ to the Plasma Physics Board}

At the end of May, the Plasma Physics Division will be electing a new Board to serve for the next three years. This will consist of 12 full members (of whom at least six cannot have served on the present Board) and up to three co-opted members, all of whom resign at the end of their term. National societies and members of the Division are invited to nominate candidates. Those from the Division should carry the signatures of three Individual Ordinary Members and an assurance that the candidate is willing to serve if elected. Proposals should be sent by 18 May 1984 to the Chairman:

Dr. A. Gibson,

JET Joint Undertaking

Abingdon, OX14 3EA, England 